\title{
AD FRIDERICUM G. KENYONEM EPISTULA EDITORIS
}

Iatricon libellus cum nune in lucem prodit quem prius salutet quam Te, vir optime, qui cum de Aristotele ipso tum de Aristotelico hoc volumine unice sis meritus. quod simulatque ante duos fere annos ex Aegypto in Museum Britannicum transvectum est, ubi nune extat numero CXXXVII, Tu quamquam multis et gravissimis negotiis paene oppressus, statim lacera fragmenta explicare, levigare, ordinare coepisti, tum seripturam et difficilem per se et saeculorum iniuria misere oblitteratam legere, conexus rimari, lacunas supplere, denique libri in dies magis crescentis materiam intellegere, aetatem constituere, fontes expiscari. quae omnia tam ineredibili celeritate ac sollertia perfecisti, ut iam mense Aprili MDCCCXCII viris doctis libri recentis formam et indolem breviter sed accurate exponere posses. ${ }^{\prime}$ iam tum recte intellexisti, vir doctissime, priorem partem voluminis, quae in antiquorum medicorum memoria continetur et iterata Aristotelis mentione est insignis, excerptam esse ex Menonis Aristotelei Iatricis. qui nuntius ut alios ita me commovit. nam cum Aristotelicis litteris novum monumentum accrescere tum Doxographorum quaestionibus olim desertis novum quasi pabulum offerri sensi. itaque quae ex Musei penu $\mathrm{T}_{E}$ quasi promo eondo prolata erant, summa me expectatione replebant. quam augebant duae prioris partis eclogae, quas Fridericus Blassius, vir eruditissimus, e Britannia redux peramice mecum communicaverat. quibus dum acrius incumbo, vidi sine totius voluminis contextu singula recte intellegi non posse. ita in dies vehementius editionem totius voluminis a VoBIs desiderare coepi. sed quaerenti mihi et instanti Tu respondisti nihil eiusmodi aut abs Te aut ab aliis parari

1 Classical Review VI (1892) p. 237-240. 
ac sero, si umquam, fore ut huic desiderio satisfieret. simul tamen, ne istius nuntii tristitiam nimis aegre ferrem, eximia benevolentia apographum Tua manu confectum in meum usum commisisti. acceptum volumen tractare, retractare, emendare, supplere meo marte eoepi, qui labor etsi difficillimus erat, tamen quo magis minui videbam fenestrarum numerum, eo maior erat in dies spes restituendi omnia. sed ne solus thesauro incubare viderer ut avarus, iam in publicum edendi consilium ortum est. quod cum Tu probasses adiutoremque operis $\mathrm{Te}$ obtulisses, venia ab E. Maunde Thompson, summo bibliothecae praefecto, viro doctissimo et humanissimo, facile impetrata institutum opus iam intentius promovere coepi, et quod privatim primo susceperam, iam Academiae nostrae auctoritate promulgare visum est, cuius Supplemento Aristotelico tam insigne monumentum deesse fas non erat.

Interim Tu nostram iam tamquam Tuam rem gerere, papyrum paene cottidie excutere, nostras coniecturas religiose conferre cum exemplari, Tus invicem supplementa mittere et acute excogitata et coram ipso teste probata. sic etsi aemulo illo studio labor incredibilem in modum procedebat, tamen amicissimi hominis patientia et benevolentia abuti videbar, si diutius Turs oculis videre pergerem. ergo postquam quae ex supplenda altera voluminis parte subnata erant studia Erasistratea et Stratouea absolvi $^{1}$, Londinum petii ac per mensem insolita caeli serenitate adiutus totam papyrum studiose perlustravi et in eius conspectu supplere quae hiabant studui. nam sic tenuissimis litterarum umbris, quae vix cerni nedum describi accuratius possint, ad restituenda verba uti licebat. hie vero etiam plenius $T_{E}$ admirabar, quantum oculis valeres ac mentis illa divinatione, quae caliginem scripturae tamquam face praetenta illustrat. sciant enim, qui talia studia non ipsi experti sunt, plurima quae nune sine ullo dubitationis signo in hac editione leguntur, plane obscura et inexplicabilia fuisse, donec sententia felici coniectura inventa simul lectionem aperiret. ergo laboris difficillima et fructuosissima pars posteriores plane lateat necesse est; nam secundus lector munitam quodammodo et expeditam viam inveniet. sic igitur ego ductus a Te sensim fallacissimae scripturae assuefactus ultro pedem proferre ausus sum. quare TuA sunt merito non solum ea quae ipse explicuisti, sed etiam quae explicare me docuisti.

1 Über das physikalische System des Straton (Sitzungsberichte der Berl. Akad. 1893 p. 101-127). 
Inveni illam papyrum vitris diligenter inclusam et in undecim tabulas commode divisam, quarum conspectum subiungo. ${ }^{1}$

Qui singulas nune in manus sumet tabulas, forsitan dubitet de or- Papyri dinis veritate, quem Tu restituisti egoque usu probatum inveni. at 'l'ıB praeter posticae seripturae indicia, de qua postea loquar, ampliora ac magis continentia fragmina voluminis olim praesto fuerunt, quae postea demum forfice sunt discissa in commodioris usus partes, quibus in diversas tabulas translatis quae olim cohaerebant dirempta sunt." etiam ceterae paginae aut nunc couiunctae servantur aut, si paucas excipias, manifesta prioris conexus retinent vestigia. sed singillatim haec perseçui is tantummodo poterit, cui vitris sublatis ipsas ehartas examinare licebit. opto vero, ut 'Tu qui huius papyri fata ab initio cognovisti, aliøuando huic desiderio satis facias.

Voluminis quantum nuuc restat longitudo, si partes contextas cogites, Ordo est trium fere metrorum ac dimidii, latitudo (altitudo) XXIII fere centesimarum. plagularum singularum, ex quibus papyri corpus conglutinatum est, commissuras vili XIX, quae quinis fere denis centesimis inter se distant. sed veri simile est paulo plures fuisse, ut paulo plus quam scapum, quem Plinius (N. H. XIII 77) vicenis plagulis constare dicit, complevisse librarius videatur. ceterum scripturam commissuris plagularum nequaquam impeditam esse omuino hodie constat et hoc exemplo confirmatur.

$$
\begin{aligned}
& 1 \text { tabula I continet paginas (columnas) } 1 \text { II III } \\
& \text { - II - - } \quad \text { - IV V VI VII } \\
& \text { - III - } \quad-\quad \text { - VIII IX X XI } \\
& \text { - IV - } \quad-\quad \text { - } \quad \text { XII XIII XIV XV } \\
& \text { - V - - } \quad \text { - XVI XVII XVIII } \\
& \text { - VI - - } \quad-\quad \mathrm{XIX} \text { et XX } \\
& \text { - VII - - - XXI XXII XXIII XXIV } \\
& \text { - VIII - . - . XXV-XXVIII } \\
& \text { - IX - - } \quad-\quad \text { XXIX XXX XXXI } \\
& \text { - } \mathrm{x} \text { - - - XXXII XXXIII XXXIV XXXV } \\
& \text { - } \mathrm{XI} \quad-\quad \text { - } \quad \text { - } \mathrm{XXXVI} \mathrm{XXXVII} \mathrm{XXXVIII} \mathrm{XXXIX}
\end{aligned}
$$

ex scissuris haec olim cohaesisse apparet extrema tabularum:

$$
\begin{aligned}
& \text { tabulae I et II coniunctae fuerunt paginis III et IV } \\
& \text { - III - IV - } \quad \text { - } \quad-\quad \text { XI - XII } \\
& \text { - V - VI - } \quad-\quad-\quad \text { XVIII - XIX } \\
& \text { - VI - VII - } \quad-\quad \text { - } \quad \text { XX - XXI } \\
& \text { - VII - VIII - - } \quad \text { - XXIV - XXV } \\
& \text { - VIII - IX - } \quad \text { - } \quad \text { - XXVIII - XXIX } \\
& \text { - IX - X - } \quad \text { - } \quad-\quad \text { XXXI - XXXII } \\
& \text { - } \mathrm{X}-\mathrm{XI} \quad-\quad \text { - } \quad-\quad \mathrm{XXXV}-\mathrm{XXXVI}
\end{aligned}
$$


Paginae (sive columnae) litteris completae servantur XXXIX, ultima semiplena; intervalla vacua, binarum fere centesimarum, extrenus tamen margo dexter, ubi librarius substitit, duplo maior. versuum numerus in singulis paginis a quadragenis quaternis sensim auctus est finem versus usque ad undesexagenos. summa versuum, computatis etiam quos supplevi versibus, sed omissis, si qui inter versus aut extra additi sunt, est MDCCCCX; quibus si aldas fragmenta posticae, fiunt MDCCCCXXVII. unde apparet (uantum reli(quum sit a iusti voluminis ambitu, cum praesertim ipsi versus legitimi stichi mensura (XV syllabarum) sint minores. nam XIII fere syllabarum (i. e. XXX fere litterarum) modulo contiuentur. initium voluminis non servatur. neque tamen ea iactura plus quam una vel duae paginae perierunt, quantum ex Prooemii superstitis natura coniectari potest. periit igitur etiam titulus libri cum auctoris nomine. quod si quis in inseriptiono IV 18.19) invenire voluerit, non invidebo; ego quid sentiam, infra p. XVIII aperiam.

Fragmenta Praeter maiora fragmenta, quae olim incerta sede fluctuabant, nunc autem omuia a 'T'E partimque a me in rectum ordinem restituta sunt, nonnulla frustula vaga servautur, quae editioni adiunxi sperans fore ut haec quoque forte fortuna aliquando corpori reddantur. his minutis fragmentis (III-XII) duo praefixi paulo ampliora, quao manu librarii in postica addita sunt. nam ut ipse liber iusto ordine i. e. in autica parte scriptus est, ubi secuudum U. WILCKENI egregiam observationem fibrarum striae cum versuum lineis eandem derectionem teneut, ita fragmenta illa in postica posita sunt, quae fibris contrariis facile agnoscitur. quae fragmenta cum pertineant sine dubio ad eam disputationem, quae in extrema pagina XXXIX non tam clauditur quam abrumpitur, cumque laborantis librarii haud obscura signa praebeant, conieci ${ }^{1}$ propterea suppleta esse, quia cum ulteriora in exemplari magis magisque corrupto legentis laborem fallerent, illa sola aliquatenus enucleari possent. etiam antea exemplaris condicionem tristiorem fuisse veri simile est, ut oblitterati apices oculis librarii vix ac ne vix quidem exciperentur. qua re excusatur aliquo modo illius incredibilis neglegentia, quae usquequaque cernitur. tot supplementa sunt inter versus et in margine, tot liturae, tot hiatus verborum et sententiarum. sed de his postea accuratius.

1 Über die Excerpte ron Menons Iutrika in dem Londoner Papyrus 137 (Hermae vol. XXVIII) p. 410. 
In postica praeter ea quae ipse librarius supplevit praeceptum me- Praceptum dici invenitur rudi calamo eoque recentiore exaratum, ut possessorem voluminis id aspersisse credas; tum scholastica exercitatio, quam in postica tabulae II aliena (saeculi tamen II p. Chr.) manu scriptam legi-

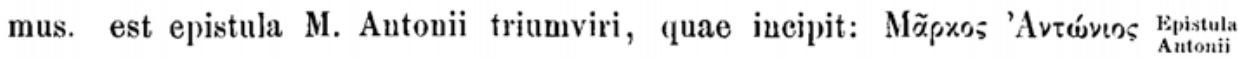

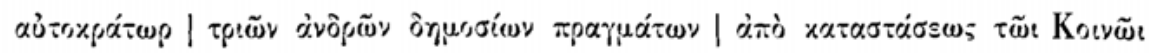

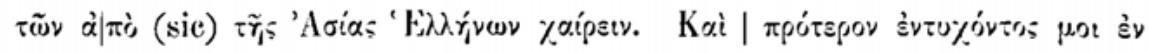

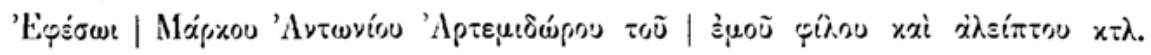
integram epistulam T\& mox editurum esse pollicitus es'. praeter haec igitur duo calamorum posteriorum pericula cetera omnia unius librarii manu perseripta sunt tam contextus verba yuam quao correcta suppletave sunt.

De aetate librarii controversia est, de qua dixi Hermue l. c. p. 411. ciararii ut enim Alexandri Philalethis crebra mentione circa Christi natalem alter terminus fixus liaeret, ita inferior vacillat. assentior tamen TUAE sententiae, vir peritissime, qui seripturam altero saceulo haud posteriorem esso asseveras." eum enim litterac sint similes primae potissimum quae exaravit vesTri Aristotelis manui, tum siglorum forma, ut idem 'Tu recte monuisti, simillina. nerque enim universa ratio diserepat et octo

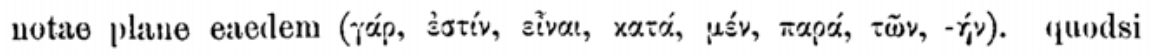
minutias formarum rimeris, etiam antiquiora videautur latricou compendia quam Aristotelea. ${ }^{3}$

Compendiorum autem in hoe volumine duo genera inveniuntur: unum compendia liberum omnibusque sedibus concessum quod siglon voeo, alterum extremis versuum vocabulis reservatum compendiornm, quae proprie dico. ae sigla quidem tabula priore huic editioni adiuncta illustravi, de compendiis vero finalibus haec habeto:

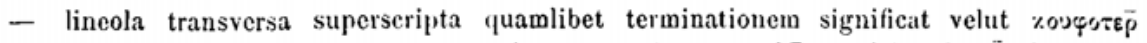

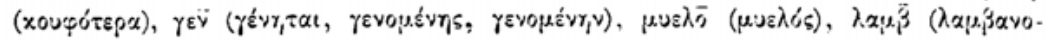

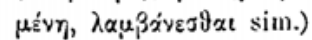

significat $r_{j}$ velut $\kappa \varepsilon \varphi \alpha \lambda-\left(x \varepsilon \varphi \alpha \lambda \eta_{j} v\right), \tau \rho \circ \varphi-\left(\tau p \circ \varphi i_{j} v\right)$

a superscriptum av

o - ov

u ou aut ous

$\omega \quad$ - $\omega s$ aut $\omega v$

1 Classical Review 1. c. p. 237.

2 Classical Review 1. c. p. 239.

${ }^{3}$ nam si derectae et simplices formae ex flexis et compositis originem duxerunt (id quod in nonnullis notis demonstrari potest ef. Lehmann Tachygraph. Abkïrzungen Lejpz. 1880 p. 102. 105), hic scriptor genere antiquiore usus est quam Aristotelis. 


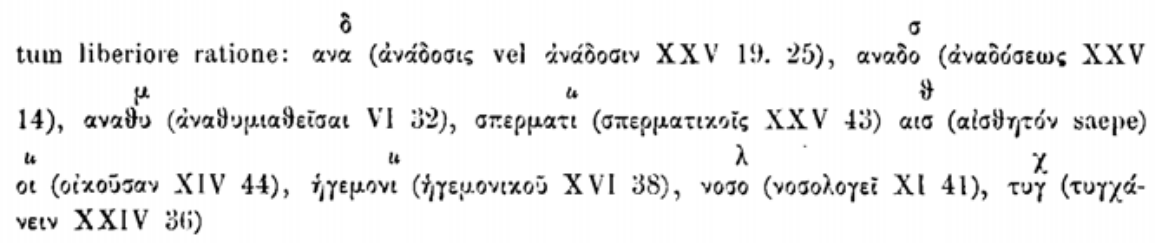

De ipsa seriptura hoc dico, quod uni euique autotypo nostro inspecto oculis videre licet, librarium non artificem fuisse libros mercede scribere consuetum, sed hominem mediocriter doctum, qui exemplar sive situ sive tritu corruptum in usum privatum describeret. eiusdem generis plerosque libros esse, qui nune in Aegypto inveniuntur, et olim dixi ${ }^{1}$ et nunc post tot tamque egregia inventa etiam confidentius assero. itaque cun duplex fuerit ut apud nostrates ita apud antiquos litteratura, una pulchra et antiqua illa titulorum, qua antiquarii qui recte dicebantur libros Sosiorum vel Atticorum iussu affabre scribebant sive pingebant, altera vulgaris et cursiva, qua vulgus epistulas, commentarios, quidquid privato usui destinatum erat, currenti calamo conscribebat sive conscribillabat: homines docti qui librum deseripturi erant cum vulgari genere uti nollent, artificioso nescirent, utrumque miscebant. talis est scriptura semicursiva eorum qui Aristotelis r. p. Atheniensium exaraverunt, talis huius hominis qui conatus est (neque enim perfecit) in suum sibi usum Iatrica baec describere. ergo litteratura ista etsi ex cursivi generis levitate adspirat ad grandius illud, tamen parum accurate et distincte formatur, ut saepe immo plerumque incertum sit, utrum $\tau$ an $v, \lambda$ an $\alpha$, $\lambda \lambda$ an $\mu, \nu$ an $\alpha$, $\gamma$ an $\tau, \alpha$ an $\varepsilon$ vel o etc. voluerit scriptor. hinc maxima legendi difficultas oritur. praeterea fluctuant ipsae formae relabente subinde librario in vulgare genus. velut praeter $x$ sollemnem formam cursivam illam $u$ non raro adhibet, ut xai an ws scriptum sit plus semel haereas. inprimis cursiva utitur in eis quae vel supra vel extra versus supplet et in compendiis finalibus. quae cum ita sint, tutissimum erat volumen totum lucis arte fideliter expressum lecturis ante oculos ponere, quod Vos, viri illustrissimi, in Aristotelis et Herodae autotypis egregio eventu fecistis. sed quod Tu mihi quaerenti olim praedixisti, re expertus sum: condicio papyri et inprimis subfuscus eius color obstant, quominus imago satis clara fiat. tamen eiusmodi autotypum editioni deesse nolui, ut scripturae saltem genus et ordo specimine cognoscantur. interim p. $7 \mathrm{sq}$.

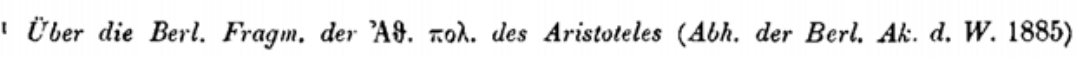
p. 7 s. 
typis, quatenus fieri poterat, papyri imaginem repraesentavi, qua in re fidei magis quam pulchritudini studuisse me confiteor. ceterum fore spero ut ex autotypo periti aetatem scripturae certius definiant. ego enim a palaeographiae scientia inprimis papyrorum, qua Te potissimum niti par est, alienum me esse sentio. video tamen eum orthographiam

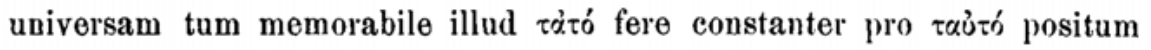
Tuas sententiae favere. nam eum morem intra duo illa saecula, quae dividuntur ut cardine Christi natali, se continere satis constare videtur. ${ }^{2}$

Orthographiae autem haec genera discribam:

$\bar{\varepsilon}$ pro $\bar{\imath}$ longa tum in usu fuisse librariis constat cum ex aliis monumentis tutn ex Ilerculanensibus voluminibus. ipsi quoque auctores huius actatis non aliter seripsisse videntur, ut recte in Philodeni editionibus talia serventur. sic hic scriba paene constanter

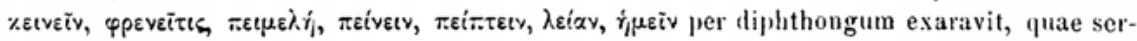

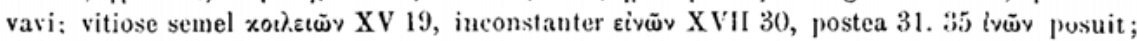

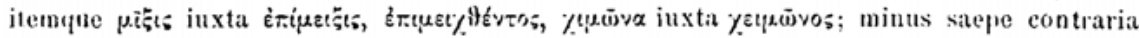

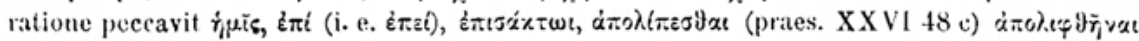
XXIII 39 alia, quae commodo magris quam rationi consulens nostro usui accommorlavi.

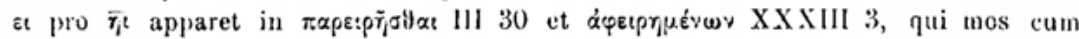
eliam in llerculauensibus voluminibus observetur ${ }^{3}$, tamquam aetatis signum servandus videtur.

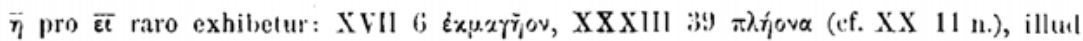
quoque primae Caesarum aetati conveniens.

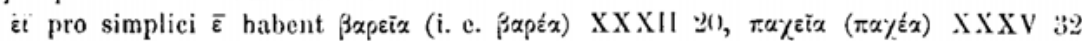
(ef. Blass-Kühner Gr. (ir. I 1, 445 n. 3).

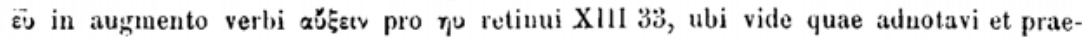
terea Blassium Ausspr. p. $44^{14}$ ).

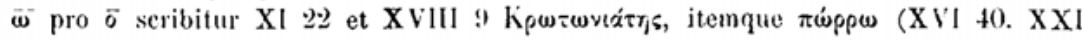

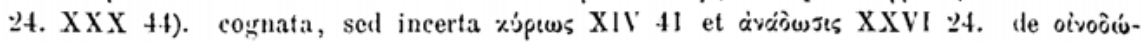

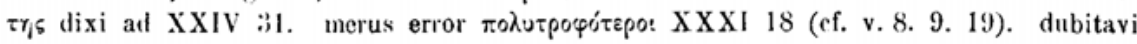

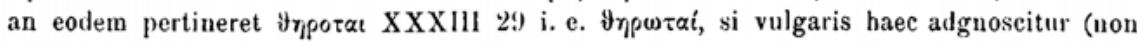
scriptoris, at librarii) forma iuxta inpeutai. quodsi haee explicatio probetur, forsitan

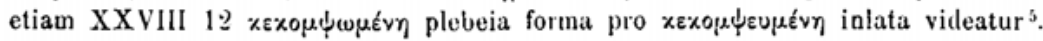

'Classical Review I. c. p. 239.

2 cf. auctoritates, quas Hermae I. c. p. $411^{1}$ attuli, et F. Blassius Ausspr. ${ }^{3}$ p. $77 \mathrm{sq}$. addo aetatis inferioris exempla ex Pap. graeca Berol. n. 6867 (anni 85 p. Chr.) quae

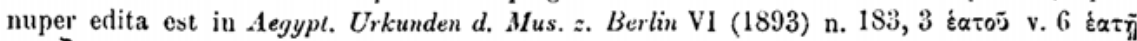
v. $29 \dot{2} \dot{\alpha} \tilde{\eta}_{\varsigma} ;$ v. 5 corrige $\dot{\varepsilon} \alpha \tau \tilde{\eta}_{\varsigma}$ (alterum documentum interiectum v. 10-27 servat formas

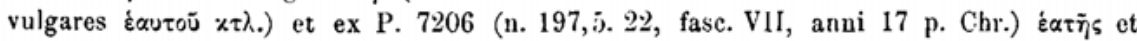

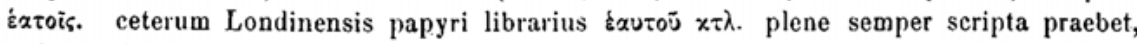
quia crasis (secundum Wackernagelii explicationem Kuhn's Z. 1893 p. 7) hic magis latet. fortasse memorabile cst XVIII 38 ATOZ i. e. átós, ó aùtós legi (superscripto tamen u).

${ }^{3}$ ef. Blass Ausspr. ${ }^{3}$ p. $46^{130}$. Mahaffy, Petrie Pap. II p. [19] B 13. [21] 3. [28] 11.

4 ef. Blass l. c. p. 59 sq.

s de his 'dicatalectis' videatur Lobeckii erudita dissertatio in Rhematico p. 199-205 et Theophrastea nostra (Berlin 1883 Progr. n. 64) p. 12 sq. 
Iota adscriptum tum plane obmutuisse omnium monumentorum testimoniis evincitur. inde vulgo omittitur non solum in privatis libris et titulis, sed etiam publicis. cuius rei lepidum exemplum exhibet Anaxenoris Magnesii titulus, qui propter litterulam illan civitati ignorantiae signum inussit. testimonium Strabonis XIV p. 648 ipso titulo

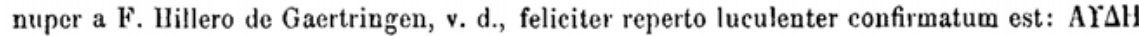
illud fauosum in lapide nune Berolinum transvecto iota caret. Iatricon librarius a Magnesiorum ueglegentia satis sibi cavit. nam in dativis fere constanter adscriptum est, at, quod mireris, eadem constantia in coniunctivis onnissum. hoc casui tribuere nemo audebit, cum praesertin idem in antiquis titulis maxime Doriensibus usu venire notum sit. post Boeckhii et inprimis Ahrensii observationes (Dial. dor. 295) res trita, nec eam ratione carere viri linguarum periti docuerunt. sed de dialecticis postea. addo in aliis velut

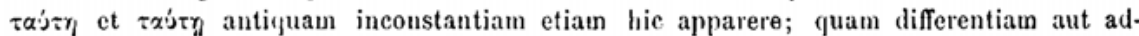
scripto aut subseripto iota sine incommodo repraesentat nostra editio.'

klisionem et crasim vocalium vitat librarius etian in particulis, sed inveniuntur

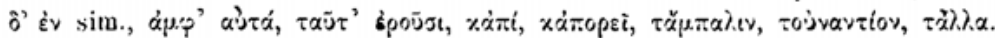

$\bar{v}$ paragogicum ut tota antiquitate ita hic quoque mira inconstantia et positum est et omissum. equidem ullam normam obtinuisse extra hiatus venatores pracfracte nego. nimirum operae pretium vix esse videbatur sonum levissimun accurate semper exprimere. ita etsi in hoc volumen cadit, ytuol in omnes antiquos seriptores, $\vee$ etiam ante consonantem saepius poni quam noster i. s. liyzautinorum posteriorum canon peruittit, tamen mira inconstantia

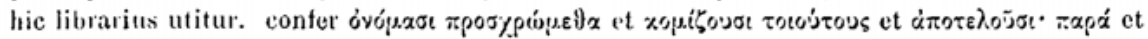

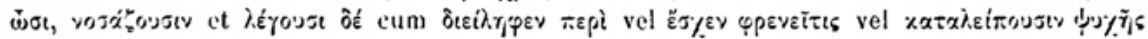

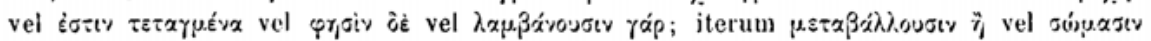

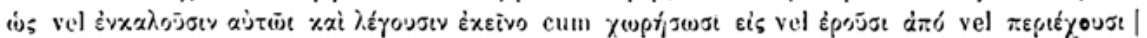

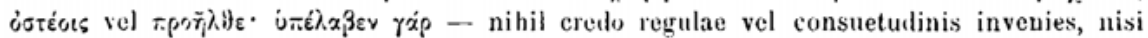

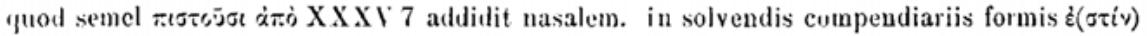
et $\varphi$ (roiv) semper plenas formas exhibui (secutus librarii exempla 111 15. VIII 12. XIV 12).

Eadem nasalis soni debilitas cernitur in aliis formis velut $\pi \alpha^{\prime}$ ! vóross VI 36 et XVIII 5 (cf. lobeckii Phry!. p. 284); set etiam in ráh. Évrotsizat XVII 20. verum haec res la-

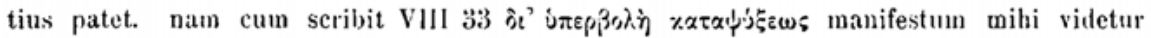

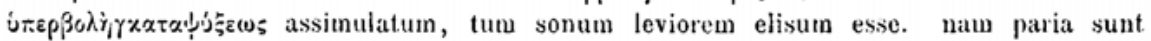

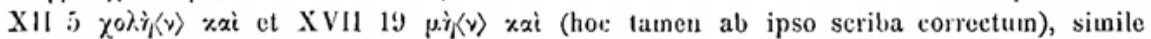

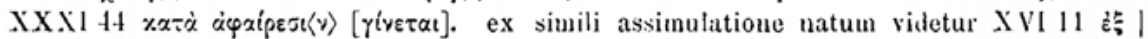

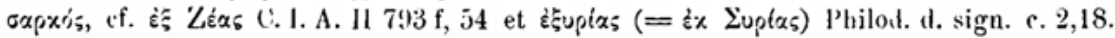

De assimulatione cum locutus sim, monendum est aequalium more interdum emolliri

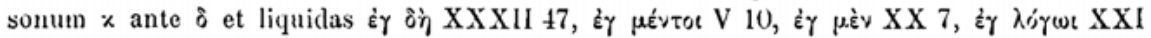

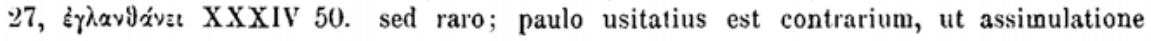

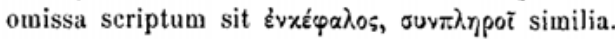

Aegyptiorum dialectum in aspiratis maxime laborare et ex papyris cognoscitur et ex Macedonicae indole facile explicatur. bic tamen scriptor ut paulo cultior homo non

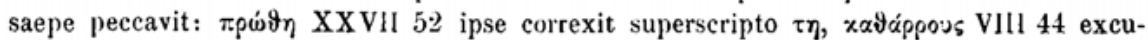

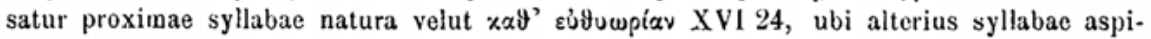

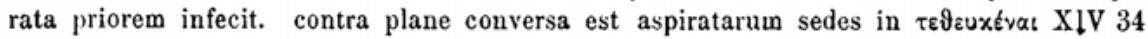

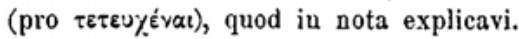

Venio ad geminorum consonantium compendia. pinguiore enim pronuntiatione per totam graecitatem factum est, ut simplici liquida efferrentur quae duplici legitime scri-

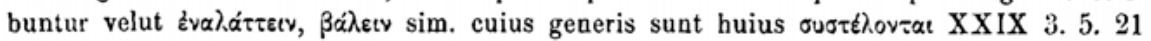

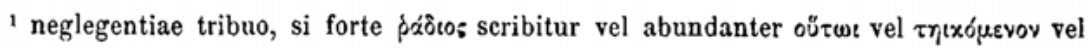

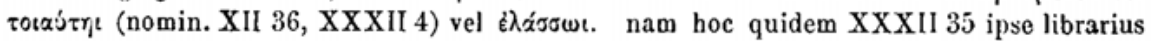

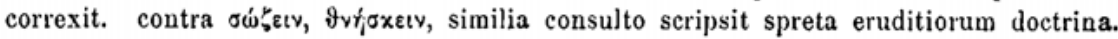


(partim correcta), porro $\mu \varepsilon \tau \alpha \beta \alpha \dot{\lambda} \varepsilon$ เv XXIV 28 (correctum), denique $\pi \varepsilon$ só $\mu \varepsilon v o v$ XXXIV 4 (quod est $\pi \varepsilon \sigma \sigma \delta \mu \varepsilon v o v)$. in $p$ soni duplicatione velut in äroppeiv antiquitus fluctuabat et pronuntiatio et orthographia. hine etsi vulgaris mos obtinet, inveniuntur tamen in hac

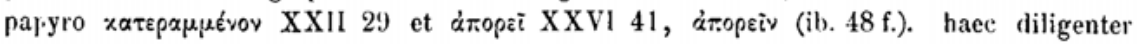
servavi, cum Aristarchus quoque simplicem $\rho$ commendaverit.

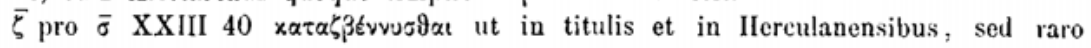
omnino invenitur.

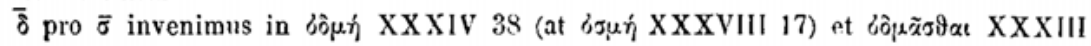
19. XXXIV 48.

Quae ultimo loco tetigi, sponte traducunt ad ionicae dialecti vestigia, Dialoctica quae ex scriptoris eiusve auctoris patria derivanda esse conieci Herm.

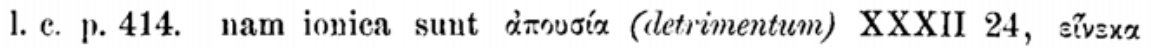

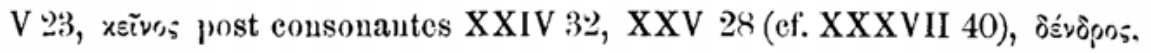

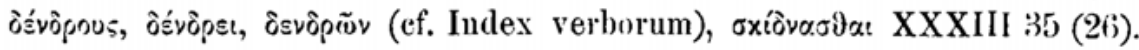
glossa (nescio cuius dialecti) est xaixi $\varsigma$ XXXVI 57 (ef. Index v.). aul

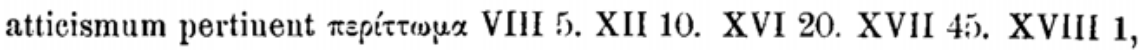

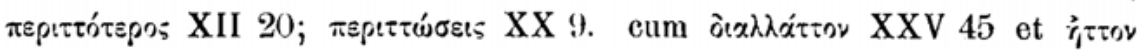
XXXVIII 40 posterioris sint partis, conici non licet atticum hune colorem ex Menoniis translatum esse, nam tenuia sunt etiam alibi vestigia atticismi velut $\hat{\omega} s$ praepositionis usus, ws ópoíws similia, de quibus in Hermae l. c. p. 412 breviter sum locutus. sed oratio in universum abhorret a cultiore

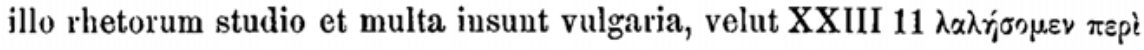

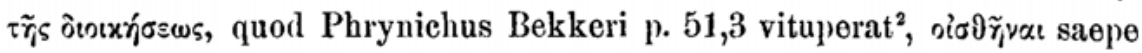

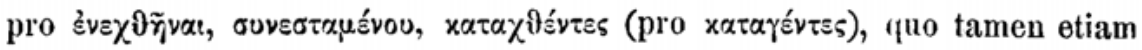

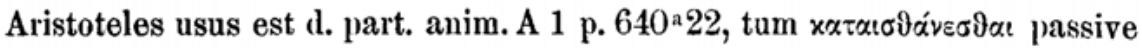
(XXXII 5), Éxqúsıy intransitive (XXV 3) et praecipue in praepositionibus et particulis, in quibus Graeculorum inscitia maxime conspicua est; cf. Index

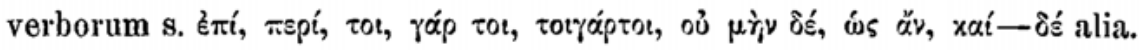

Interpunctum est a librario diligentius quam aequales consueverant. Interpunctio quattuor enim invenias distinguendi genera. primum crasso puncto finis imponitur periodo XXXI 25, quod est singulare. legitime autem spatia vacua adbibentur, aut minutum (i. e. $*$ huius editionis), quo membra periodi sive enuntiata minora dirimuntur, aut amplius (i. e. **), quo maiora enuntiata vel periodi finiuntur. huic autem spatiorum signo, quod est inter versus, respondet duplex item extra versus positum a sinistra. nam initio versuum minore spatio (*) diremptorum praetexi solet paragraphus obelo similis ( $\longrightarrow$, itemque maioribus spatiis respondet diple $(-)$.

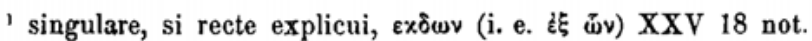

af. Apollodorus Poliorcet. p. 137, 4 i $\pi \varepsilon \lambda \alpha^{2} \lambda \eta_{j} \sigma \alpha$ (sub Hadriano).
} 


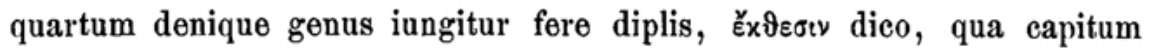
singulorum initia litteris versuum primoribus sinistrorsum expositis facilius in legentium oculos cadant. ${ }^{1}$ ceterum totius rationis (quam utinam librarius constantius adhibuisset) quanta sit utilitas ad libri sententias sive intellegendas sive supplendas suapte sponte intellegitur. quare huic rei nobis maxima impensa est cura.

Etiam illud magnopere interest eorum qui supplendi negotium suscepturi sint, ut versiculorum exitus sedulo notentur, cum praesertim in hac sede liberiore compendiorum genere usus sit librarius. omnino hoc tenendum est, quod non semper recte ab editoribus observatum vidi, inde ab Alexandrina aetate versiculos dirimi syllabatim. quod, etsi nusquam usus est in fugam vacui angulo illo, qui in Hyperideis et Herculanensibus voluminibus conspicitur, accurate tamen semper librarius obser-

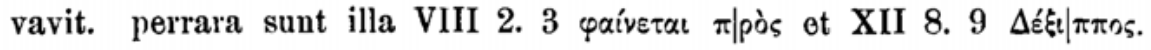

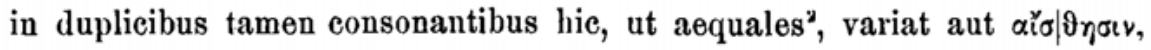

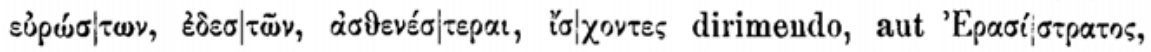

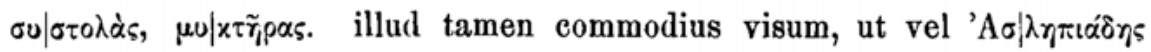
cum seripsisset, $x$ post $\sigma$ addere quam $\lambda$ litterae praescribere mallet.

Morem indolemque librarii scrutanti non omittenda est ea neglegentia, quae in omittendis litteris, syllabis, verbis, enuntiatis versatur. quae partim ipse correxit (neque enim quisquam praeter eum diorthotae munere functus est), partim nobis supplenda reliquit. atque illa quidem

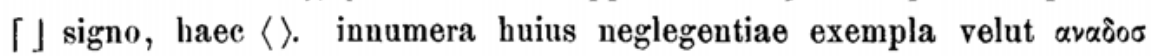

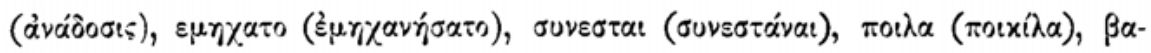

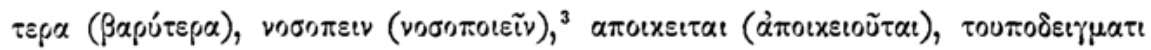

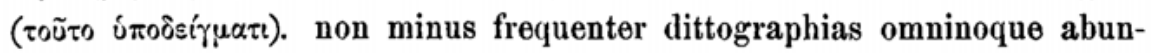
dantias commisit. et dittographiarum quidem prius vocabulum delere

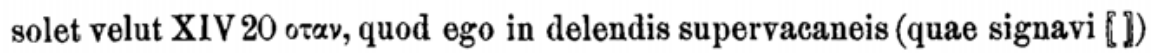
secutus sum. delendi autem tria genera adbibet: lituram digitis madidum atramentum abstergentibus factam, puncta superposita, lineam transver-

1 quartum hoc genus in membranis inde ab antiquissimis quinti saeculi usque ad XIII interdum inveniri notum est (cf. Gardthausen Palaeogr. p. 275/6). ipse nuper eiusmodi codicem usurpavi Pbilonis Academiae Petersburgensis XX A a 1 chart. s. XIII/XIV fol. min., ubi post capitis exitum versus proximi initium rubro exponi solet.

${ }^{2}$ cf. Blassius ad Hyperidem p. IX.

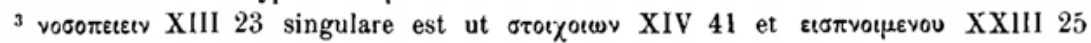

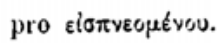


sam. memorabile mihi videtur singulare abundantiae genus, quod nescio an ad indolem hominis pernoscendam faciat plurimum. ut si legimus

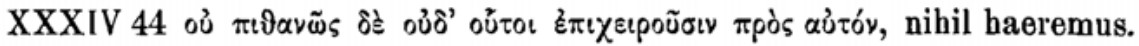
at delevit ipse librarius $\pi$ poss aưóv, scilicet quia non erat in exemplari,

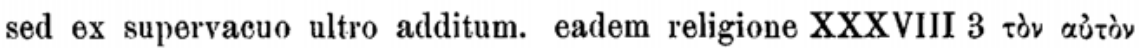

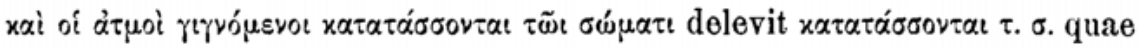
e loci contextu ariolatus contra exemplar addiderat; et sic saepe.

Talia non sunt operarii vix sensum curantis et digito syllabas $\begin{gathered}\text { Lilirarii } \\ \text { indoles }\end{gathered}$ exemplaris carpentis, sed paulo liberioris hominis, qui tota enuntiata excipere et extrema deficiente memoria cogitando supplere consueverit. itaque exarasse haec crediderim adulescentem aliquem medicinae studiosum, qui in suum sibi usum haue isagogen describeret. de medicinae eius scientia nolo quidquam detrahere, quamquam caput et ventrem confundere (VIII 14) non cuiusvis est. at in philosophia plane hospes

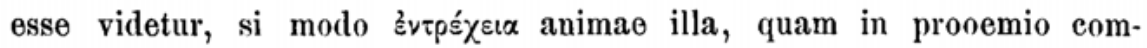

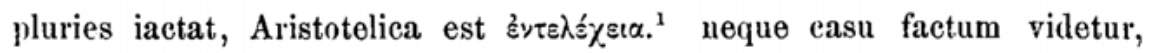
quod ubi iterum animae mentio iniecta est XXXI 42 sqq., filum disputationis interrumpitur. scilicet librarii scribunt, ut ait Hierouymus, non quod inveniunt, scd quod intellegunt. obscuratis igitur exemplaris apicibus interdum intellegentiam eum defecisse consentaneum.

De auctoris nomine, secta, aetate, fontibus accuratiora ut invenirem, Auctor ne nunc quidem contigit. quid mihi visum sit, significatum est alias ${ }^{2}$. hoc omni dubitationi exemptum est post Alexandrum Philalethem Laodicensem eum seripsisse i. e. primo p. Chr. saeculo (si modo exempli Londinensis aetas recte supra definita est) ac praeter hunc Menonis Iatrica, quae sub Aristotelis nomine ei ut aliis innotuerunt, ad doxographiam prioris partis (c. IV 31-XXXI 9) adsumpta esse.

Iatricon Menoniorum fragmenta ex V. Roser, viri doctissimi, syllogis Menonia Berolinensi ${ }^{3}$ et Lipsiensi ${ }^{4}$ transtuli (paucis tamen mutatis), ut imago tanti operis, de quo Londinensis papyri beneficio iam accuratius edocemur, quam plenissima restituatur. nam si latina fragmenta (IV-VII)

\footnotetext{
1 Hermae 1. c. p. 411.

${ }^{2}$ ef. Hermae l. c. p. 413 sq.

${ }^{3}$ Aristotelis opera ed. Ac. R. Bor. V (1870) p. 1534 n. $335-341$.

4 Aristotelis fragmenta Lips. 1886 p. 255 sqq. n. $373-379$.
} 
ut parum certa omittas, ex Plutarchi tamen loco (fr. III) satis constat non solum principia morborum, quibus contentus fuit hic scriptor, sed etiam ipsorum morborum varietatem, doxographi nimirum ratione, persecutum esse Menonem. quid? si eidem fr. (conlatis praesertim fr. IV. V) fidas, videtur Menon placitorum enarratioui definitiones singulorum morborum praemisisse, quod neque dedecet Peripateticum philosophum et similiter invenitur in Doxographis. vide Aëtii Placita I 1. 2. 9 sqq., quorum definitiones etsi colorem habent recentiorem, morem tamen traxisse videntur e schola Theophrastea, quemadmodum Characterum defiuitiones noviciae non sine exemple antiquo scriptae mihi esse videntur.

De titulo libri quae exposui Hermae l. c. p. $407^{2}$ hic repetere non inutile credo. Laertii index V 25 in Aristotelis operibus recenset 'l $\alpha \tau p$ x d̀

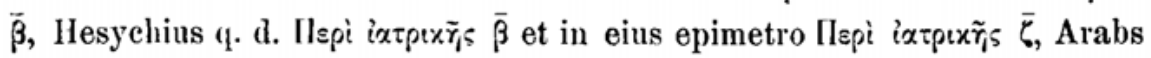
(qui vita Ptolemaei Chenni usus vicletur) n. 92 Пspi i $\alpha \tau p \iota x \tilde{y}_{i} \varsigma$ sine librorum numero. medici latini eitant Aristotelem(fr.IV_-VII)utDemetrius Magnesius (fr. II) et anonymus Londinensis. contra Plutarchus (fr. III) Mevóveı $\alpha$, et Ga-

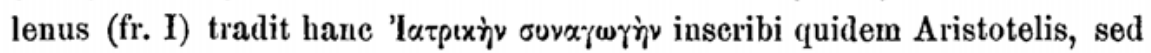
esse Menonis, Aristotelis discipuli; quare a nonnullis Mevóveı appellari.

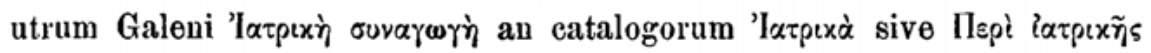

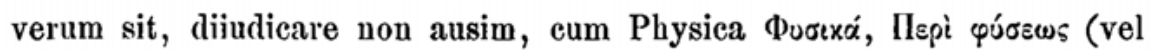

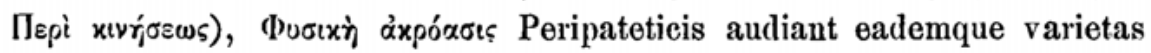
in ceteris scriptis et apud ipsum Aristotelem appareat. sed Mevóvsıa sic appellata esse arbitror ut Eỏônusı et Nıxoú́xєıа (nam ea quoque Nicomachi auctoris credebantur), quibus si Aristotelis auctoritas addebatur, doctrinam ubique apparere Aristoteleam significabatur. scilicet Stagiritae magnum nomen obumbrat, etsi num quid praeter ipsum consilium et rationem colligendi Aristoteles impertiverit Menoni, dubium mihi esse videtur.

Sed quomodocumque ea de re iudicandum erit, gratulamur nobis, qui gravissimi certe operis iam non umbram solum, sed solida fragmenta teneamus. quae etsi compilatorum culpa pristino colore privata sunt nec magnis ipsius Menonis erroribus carent ${ }^{1}$, tamen campum diu neglectum, historiam dico medicorum graecorum, novo et insperato eruditionis flumine laetificarunt. p. $424-434$.

1 inprimis de Hippocratis scriptis egregie fallitur, ut exposui Hermae 1. c. 
T'ibi vero, Friderice Kenyon, eum finem facio praefandi, pro egregiis Turs beneficiis debitam meritamque gratiam persolvo. si suavissima TUA laboris societate diutius uti et quot menses, tot annos mihi impendere licuisset, perfectius et maturatius iam opus proditurum esse probe scio. at exemplum celeritatis aţue strenuitatis, gnod 'Tu in Aristotele et Heroda proposuisti, mihi sequeudum esse putavi, non solum quia nova quam velocissime efferri omnium virorum doctorum interest, sed quia commodatam guasi nee propriam rem mo tenere existimari, quam reddere quanto ocius fas est. quod dum facio, si quid hac festinatione vel omissum vel delietum est, consoletur nos illud Senecae: contenti simus incentis, aliquil teritati at posteri conferent.

Berolini 1d. Sept. MIDCCCLXXXXIII. Hermannus Diels. 